\title{
Fluid substitution in rocks saturated with viscoelastic fluids
}

\author{
Dina Makarynska ${ }^{1}$, Boris Gurevich ${ }^{2}$, Jyoti Behura ${ }^{3}$, and Mike Batzle ${ }^{4}$
}

\begin{abstract}
Heavy oils have high densities and extremely high viscosities, and they exhibit viscoelastic behavior. Traditional rock physics based on Gassmann theory does not apply to materials saturated with viscoelastic fluids. We use an effective-medium approach known as coherent potential approximation (CPA) as an alternative fluid-substitution scheme for rocks saturated with viscoelastic fluids. Such rocks are modeled as solids with elliptical fluid inclusions when fluid concentration is small and as suspensions of solid particles in the fluid when the solid concentration is small. This approach is consistent with concepts of percolation and critical porosity, and it allows one to model sandstones and unconsolidated sands. We model the viscoelastic properties of a heavy-
\end{abstract}

oil-saturated rock sample using CPA and a measured frequencydependent complex shear modulus of the heavy oil. Comparison of modeled results with measured properties of the heavy-oil rock reveals a large discrepancy over a range of frequencies and temperatures. We modify the CPA scheme to account for the effect of binary pore structure by introducing a compliant porosity term. This dramatically improves the predictions. The predicted values of the effective shear modulus of the rock are in good agreement with laboratory data for the range of frequencies and temperatures. This confirms that our scheme realistically estimates the frequency- and temperature-dependent properties of heavy-oil rocks and can be used as an approximate fluid-substitution approach for rocks saturated with viscoelastic fluids.

\section{INTRODUCTION}

Growing demand for hydrocarbon energy sources has renewed interest in production from bituminous and heavy-oil reservoirs. Heavy-oil reserves account for more than 6 trillion barrels-in-place worldwide - triple the world's reserves of conventional oil and gas (Batzle et al., 2006). However, the high viscosity of heavy oils makes them difficult or impossible to produce using conventional technology. A variety of methods currently used by the industry aim at lowering the viscosity by injecting heat or chemical solvents. Thermal recovery methods have been the most efficient in heavy-oil production, with recovery rates as high as $80 \%$. Injected heat alters the already-complex physical properties of the heavy-oil reservoirs, such as seismic velocity, density, and attenuation. Tracking these changes with time-lapse seismic techniques can considerably improve recovery efficiency. Appropriate rock-physics modeling is important for quantitative interpretation of the changes in seismic re- sponse due to alterations in reservoir and fluid properties during production.

Seismic fluid-substitution modeling using Gassmann's equation (Gassmann, 1951) is routine in analyzing and interpreting seismic velocities and amplitudes (Smith et al., 2003). Given pore, frame, and fluid properties of a rock, Gassmann's equation can be used to predict the bulk modulus of a rock under quasi-static conditions. The corresponding dynamic moduli can be obtained from Biot's theory of poroelasticity (Biot, 1956), an extension of Gassmann's theory to finite frequencies. One important result of the Gassmann/Biot theory is that the effective shear modulus is identical to the frame shear modulus. In other words, the existence of the fluid has no effect on the effective shear modulus of the rock.

As known from laboratory measurements (Nur et al., 1984; Eastwood, 1993; Schmitt, 1999; Batzle et al., 2006; Behura et al., 2007; Han et al., 2007a), heavy oils exhibit viscoelastic behavior such that their moduli are frequency and temperature dependent. Oil that be-

Manuscript received by the Editor 18 August 2009; published online 9 April 2010.

${ }^{1}$ Curtin University of Technology, Department of Exploration Geophysics, Perth, Australia. E-mail: d.makarynska@curtin.edu.au.

${ }^{2}$ Curtin University of Technology, Department of Exploration Geophysics and CSIRO Division of Petroleum Resources, Perth, Australia. E-mail: b.gurevich@curtin.edu.au.

${ }^{3}$ Formerly Colorado School of Mines, Department of Geophysics, Center for Wave Phenomena, Golden, Colorado, U.S.A.; presently BP Americas, Exploration and Production Technology, Houston, Texas, U.S.A.E-mail: jyoti.behura@bp.com.

${ }^{4}$ Colorado School of Mines, Department of Geophysics, Center for Rock Abuse, Golden, Colorado, U.S.A. E-mail: mbatzle @ mines.edu.

(c) 2010 Society of Exploration Geophysicists. All rights reserved. 
haves like a Newtonian liquid at low frequencies can act as a nearly elastic solid at high frequencies. In between these extreme cases, wave propagation in heavy oil is dispersive and exhibits strong attenuation. Indeed, the heavier the oil, the more long-chain or highcarbon-number molecules present, resulting in higher viscosity. Although viscosity is influenced by pressure and gas content, it is primarily a function of oil gravity, composition, and temperature. Depending on temperature conditions, heavy oil can have a finite shear modulus even at seismic frequencies, making Gassmann/Biot's poroelastic constitutive equations inapplicable to heavy-oil rocks.

When a rock is saturated with heavy oil, its behavior also becomes viscoelastic (Behura et al., 2007). A dramatic decrease of compressional velocities in heavy-oil sands with increasing temperature has been observed in laboratory experiments (Tosaya et al., 1987). Despite the fact that this decrease was in part caused by the abnormally high pressure in the experimental setup, the main factor responsible for the velocity decrease is change in fluid properties with temperature.

Laboratory experiments provide evidence of the complex properties of heavy-oil rocks. In particular, Han et al. (2007a, 2007b, 2008) study various effects on the compressional- and shear-wave velocities of heavy-oil sands, such as rock texture, pore-fluid properties, pressure, and interaction between pore fluids and rock-frame properties at different temperatures. Han et al. (2007b) also test Gassmann predictions against experimental data for a range of temperatures. They indicate that as temperature decreases below $60{ }^{\circ} \mathrm{C}$, heavy oil becomes dispersive and Gassmann estimates no longer match the data. Viscoelastic behavior of the oil violates Gassmann theory. Indeed, Gassmann theory is based on Pascal's Law, which states that, in the absence of body forces, fluid pressure is the same throughout the pore space. This law is not applicable to viscoelastic media. Biot's theory is not applicable to heavy oils either because it ignores the viscoelastic phenomenon by neglecting the fluid shear stress in the microscopic (pore-scale) constitutive equations (Pride et al., 1992; Gurevich, 2002).

Several approaches to modeling effective elastic properties of rocks saturated with heavy oil have been proposed (Marion and Nur, 1991; Eastwood, 1993; Tsiklauri and Beresnev, 2003; Leurer and Dvorkin, 2006; Ciz and Shapiro, 2007; Das and Batzle, 2008; Gurevich et al., 2008). However, verification of these models using controlled laboratory experiments was not possible. We propose an alternative modeling scheme based on equivalent-medium theory for rocks saturated with viscoelastic fluids. Comparison with laboratory data allows us to refine the scheme using the concept of dual porosity to obtain reasonable agreement between theory and experiment. Our approach can be used for practical applications to produce reliable estimates of effective frequency- and temperature-dependent properties of heavy-oil rocks.

\section{METHODOLOGY}

\section{Effective-medium model}

Fluid saturation is an important parameter influencing the seismic properties of rocks. As mentioned, the effect of a Newtonian pore fluid on the elastic moduli of a rock can be predicted from the properties of dry rock and fluid compressibility using the Gassmann/Biot theory. An alternative approach, which addresses the problem of estimating elastic moduli from knowledge of rock constituents and microstructure, is based on effective-medium theories (e.g., Hashin and Shtrikman, 1963; Budiansky, 1965; Hill, 1965; Mukerji et al.,
1995; Kazatchenko et al., 2004). These theories allow one to compute equivalent elastic properties of a mixture of two or more elastic constituents. Unlike the Gassmann/Biot theory, effective medium theories require characterization of matrix and pore space geometry. Furthermore, at sufficiently low frequencies, these theories (schemes) can be applied to mixtures of viscoelastic constituents by considering them as solids with frequency-dependent moduli. This is known as elastic-viscoelastic analogy or the correspondence principle (Hashin, 1970; Haddad, 1995). Hence, effective-medium theories can be applied to heavy-oil rocks.

To gauge the effect of viscoelasticity of the pore-filling material on the overall rock properties, we use Berryman's (1980, 1992) scheme based on a self-consistent theory. This scheme is a self-consistent version of the average T-matrix approximation of Küster and Töksöz (1974) and is also known as coherent potential approximation (CPA). CPA is a versatile mixing law of the theory of composites. It is known to provide reliable estimates of frame moduli of heterogeneous materials (Ogushwitz, 1985). CPA uses the concepts of elastic-wave scattering theory for the deformation of isotropic inclusions and approximates the interaction of the inclusions by replacing the background medium with an as-yet-unknown effective medium. This means that the effect of many pores of a particular shape is given by solving the canonical problem of a single pore surrounded by a uniform medium with yet-unknown elastic properties of the porous composite. Milton (1985) proves rigorously that CPA is a realizable effective medium scheme, i.e., there exists a particular geometric arrangement of the constituents for which this scheme is exact.

A key property of CPA is that it is symmetrical with respect to the constituents. Each constituent is treated equally in the scheme, meaning that no single constituent acts as a host to the others but that a more abundant constituent is the load-bearing one. Thus, a solidfluid mixture is modeled as a solid with fluid inclusions of a particular shape when fluid concentration is small and as a suspension of solid particles in the fluid when the solid concentration is small. This property is consistent with the concepts of percolation and critical porosity (Nur et al., 1995) and allows one to model situations when heavy oil is a pore-filling fluid and when it is part of the rock matrix.

CPA calculates the effective properties of a porous rock using known properties of the solid matrix, the pore fluid, and the pore aspect ratio. The implicit CPA formulas for bulk $K$ and shear $G$ moduli of a two-component rock (one solid phase, one fluid phase) are

$$
\begin{gathered}
\phi\left(K_{f}-K\right) P^{f}+(1-\phi)\left(K_{s}-K\right) P^{s}=0, \\
\phi\left(G_{f}-G\right) Q^{f}+(1-\phi)\left(G_{s}-G\right) Q^{s}=0,
\end{gathered}
$$

where $\phi$ is porosity; $K_{f}$ and $G_{f}$ are bulk and shear moduli of the pore fill, respectively; $K_{s}$ and $G_{s}$ are bulk and shear moduli of the matrix (grain) material, respectively; and $P$ and $Q$ are invariants of the $\mathrm{Wu}$ tensor (Wu, 1966). The components of this tensor depend on the aspect ratio of the pores and on the bulk and shear moduli of the pore fill, matrix material, and as-yet-unknown effective moduli $K$ and $G$ of the composite. We use the expressions of the Wu tensor for spheroidal inclusions of arbitrary aspect ratio $\alpha$ to describe the pore/grain geometry of typical sandstones. These expressions are cumbersome and can be found in Berryman (1980) or Mavko et al. (1998).

Equations 1 and 2 are coupled and can be solved by iteration:

$$
K_{n+1}=\frac{\phi K_{f} P_{n}^{f}+(1-\phi) K_{s} P_{n}^{s}}{\phi P_{n}^{f}+(1-\phi) P_{n}^{s}},
$$




$$
G_{n+1}=\frac{\phi G_{f} Q_{n}^{f}+(1-\phi) G_{s} Q_{n}^{s}}{\phi Q_{n}^{f}+(1-\phi) Q_{n}^{s}} .
$$

Equations 3 and 4 can be used to estimate the effective elastic moduli of a saturated rock. The iteration process requires a first guess of $K_{1}$ and $G_{1}$, which can be calculated using the Voigt-Reuss-Hill average. The scheme can also be used to calculate the dry-frame properties $\left(K_{\mathrm{dry}}, G_{\mathrm{dry}}\right)$ by setting the bulk and shear moduli of the saturating fluid to zero (rock with empty pores).

\section{Complex shear modulus of heavy oil}

To apply CPA to calculate the effective properties of a rock saturated with heavy oil, we need to know its complex temperature- and frequency-dependent shear modulus. For viscoelastic materials, the shear modulus $G$ is complex and represents the ability of a material to store energy elastically and to dissipate it as a viscous fluid:

$$
G=G^{\prime}+i G^{\prime \prime}
$$

where $G^{\prime}$, in viscoelasticity theory, is a storage modulus and $G^{\prime \prime}$ is a loss modulus (Ferry, 1980).

The frequency dependency of the complex shear modulus of heavy oil can be approximated by Cole-Cole empirical dispersion equations (Cole-Cole, 1941; Gurevich et al., 2008), which relate complex shear modulus with shear moduli at the low- and high-frequency limits, angular frequency, and relaxation time. The relaxation time depends on fluid viscosity, which is a function of temperature. According to Han et al. (2008), the temperature dependency of heavy oils can be described by three main stages, depending on the oil liquid point: liquid state, quasi-solid state, and solid state. This dependency can also be approximated by empirical relationships (e.g., Beggs and Robinson, 1975).

The dependency of properties of linear viscoelastic materials on frequency and temperature can be simplified by using the temperature-frequency superposition principle, which states that the dispersion curves for different temperatures are the same if the frequency is normalized by some temperature-dependent parameter (Williams et al., 1955). This means, for instance, that the Cole-Cole dispersion equation can be used for all temperatures using the Beggs and Robinson (1975) viscosity-temperature relationship.

To perform fluid substitution for heavy-oil rocks, frequency- and temperature-dependent complex modulus must be used in equations 3 and 4 instead of $G_{f}$.

\section{Comparison with known solutions for dry and liquid-saturated rocks}

Before applying CPA to heavy-oil rocks, we test its applicability to the limiting case of Newtonian liquid/solid mixtures. Figure 1 shows CPA predictions for dry and water-saturated rocks with a quartz matrix for a range of porosities and aspect ratios. The predicted bulk moduli are compared with the empirical model of Krief et al. (1990) and the Hashin-Shtrikman (HS) bounds (Hashin and Shtrikman, 1963). The Krief et al. estimates of $K_{\text {dry }}$ correspond more closely to the CPA estimates with $\alpha=0.2$, which are consistent with values of aspect ratios for sand-related pores reported in literature (e.g., Xu and White, 1995). As expected, all CPA estimates lie within HS bounds.

In Figure 2, we compare the difference between CPA estimates of saturated and dry moduli $\left(K-K_{\text {dry }}\right)$ with Gassmann predictions for quartz/water mixtures for realistic crack densities $\varepsilon<0.5$ (crack density is related to porosity $\phi$ and aspect ratio $\alpha$ of the spheroidal crack by $\varepsilon=3 \phi / 4 \pi \alpha)$. This comparison is important because any mixture model for rock saturated with a viscoelastic substance must be consistent with Gassmann equations when the pore-filling substance is a Newtonian fluid. At the same time, models based on isolated ellipsoidal pores are not exactly Gassmann consistent because fluid pressure in the pores varies with their orientations (Thomsen, 1985; Mavko and Jizba, 1991). As can be seen from Figure 2, the CPA results are in the best agreement with Gassmann predictions for spherical pores and show the maximum deviation from Gassmann estimates for relatively flat pores. For aspect ratios $\alpha=0.2-0.4$ and typical porosities of up to 0.25 , we observe satisfactory agreement with Gassmann predictions. The discrepancy is less than $1 \mathrm{GPa}$. We thus conclude that CPA is approximately Gassmann consistent within typical measurement errors.
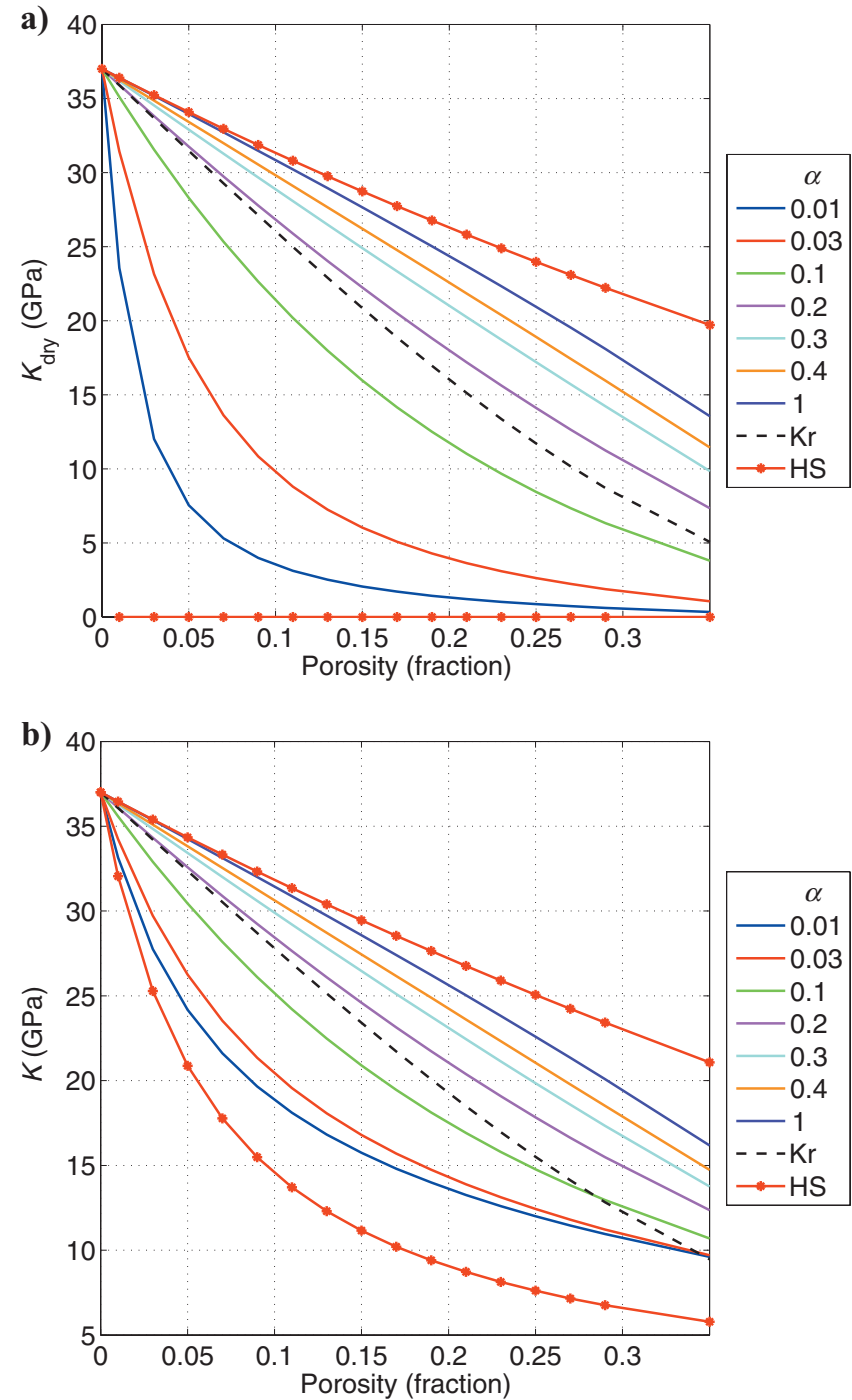

Figure 1. Bulk moduli of dry (a) and saturated (b) quartz sandstone versus porosity. CPA estimates for different aspect ratios $\alpha$ (solid lines), Hashin-Shtrikman bounds (symbols) and Krief et al. model (dashed lines). Comparison of CPA estimates of (a) dry bulk modulus of a rock with quartz matrix and (b) bulk modulus of a water/ quartz mixture. Hashin-Shtrikman bounds (HS) and Krief et al. model $(\mathrm{Kr})$ for different aspect ratios and porosities. 


\section{FLUID-SUBSTITUTION SCHEME USING CPA}

If the effective pore aspect ratio $\alpha$ is known, the effective elastic properties of a rock can be computed by iterating equations 3 and 4 . Here, we assume that the rock structure can be approximated by ellipsoids with the same effective aspect ratio. Of course, pore geometry of real rocks is more complicated and consists of pores of different sizes and shapes. However, ellipsoids often are used to capture some essential properties of subsurface voids, providing simple parameterization of the enormous complexity of the real pore space.

For a given rock sample, the effective pore aspect ratio $\alpha$ can be estimated from dry moduli (in Gassmann fluid substitution, $K_{\text {dry }}$ is the key input parameter as well). Given $K_{\text {dry }}$, the effective aspect ratio $\alpha$ can be estimated by the inverse CPA scheme (equations 3 and 4) with fluid moduli set to zero. The aspect ratio $\alpha$ can also be derived using the inverse CPA scheme if we know the bulk modulus $K$ of the rock saturated with a fluid with known bulk modulus (e.g., water). Alternatively, aspect ratio can be estimated from shear moduli.

Once $\alpha$ is estimated, we can calculate the effective moduli of a saturated rock for any pore-filling material using the forward CPA scheme. The CPA fluid-substitution workflow involves three steps. First, inverse CPA is used to estimate the effective $\alpha$ using known dry or saturated bulk or shear modulus. Second, we obtain the frequency- and temperature-dependent complex shear modulus of the pore fill $G_{f}$ from laboratory measurements. If such measurements are not available in the range of temperatures and frequencies required, they can be interpolated using known empirical relationships (Cole-Cole, 1941; Beggs and Robinson, 1975) and the frequencytemperature superposition principle (Williams et al., 1955; Ferry, 1980). Finally, we calculate the effective elastic moduli of a rock saturated with viscoelastic fluid using the forward CPA scheme (equations 3 and 4) with the complex shear modulus of pore fill $G_{f}$.

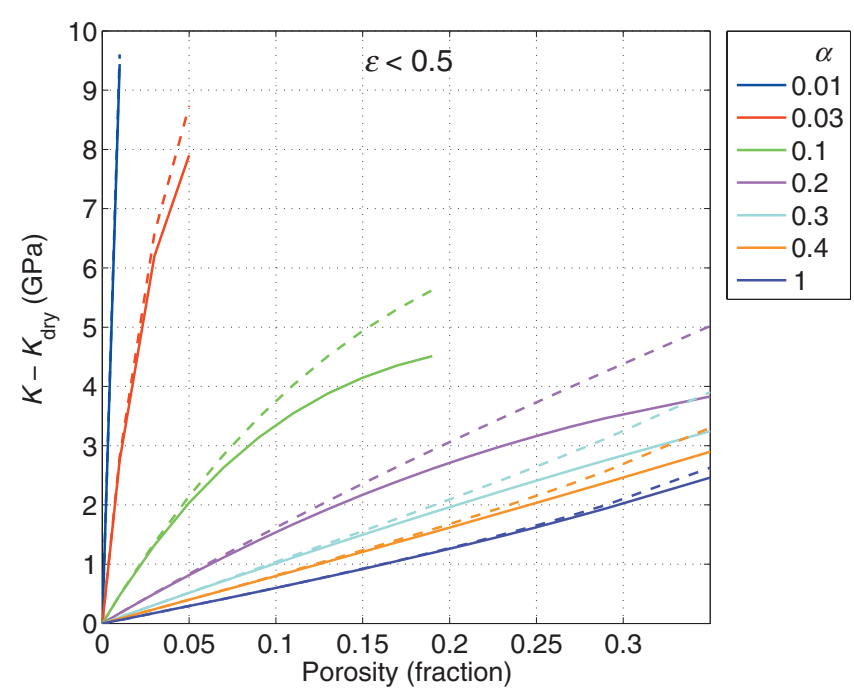

Figure 2. Difference between water-saturated and dry moduli of a rock with quartz matrix as a function of porosity and aspect ratio $\alpha$ for crack densities $\varepsilon<0.5$ : CPA (dashed lines) versus Gassmann estimates (solid lines).

\section{COMPARISON WITH LABORATORY MEASUREMENTS}

\section{Uvalde heavy-oil rock}

We apply our CPA fluid-substitution scheme to Uvalde heavy-oil rock from Texas, U.S.A. The laboratory measurements conducted on this rock have been reported in the literature (Batzle et al., 2006; Behura et al., 2007; Das and Batzle, 2008).

The Uvalde rock is a carbonate saturated with extremely viscous heavy oil with an API density of five $\left(1.12 \mathrm{~g} / \mathrm{cm}^{3}\right)$. It has porosity of approximately $25 \%$, and permeability of $550 \mathrm{mD}$. A shear rheometer was used to measure the complex shear modulus of the rock at temperatures ranging from $30^{\circ} \mathrm{C}$ to $350^{\circ} \mathrm{C}$ and frequencies ranging from 0.01 to $80 \mathrm{~Hz}$. Then the oil was extracted and its complex shear modulus was measured in the same frequency range and temperatures ranging from $30^{\circ} \mathrm{C}$ to $250^{\circ} \mathrm{C}$. The measured storage modulus of the Uvalde rock and the extracted oil for the range of frequencies and temperatures ranging from $30^{\circ} \mathrm{C}$ to $200^{\circ} \mathrm{C}$ are shown in Figure 3. The missing data points have been interpolated. The modulus of the heavy-oil-saturated rock shows a moderate dependency on frequency and a strong influence by temperature. The storage modulus $G^{\prime}$ of the rock increases with frequency for temperatures less than $150^{\circ} \mathrm{C}$. At higher temperatures, the behavior of the storage modulus $G^{\prime}$ is weakly dependent on frequency.

The trends observed for the rock shear properties look similar to those for the extracted oil. The Uvalde oil shows noticeable dispersion for temperatures less than $180^{\circ} \mathrm{C}$. At low temperatures, the extracted heavy oil supports a shear wave; but with increasing temperature, its storage modulus decreases rapidly, similar to what is observed for the rock shear modulus. As in the case with the rock, at higher temperatures the storage modulus of the oil $G_{f}^{\prime}$ is nearly independent of frequency. In general, $G_{f}^{\prime}$ increases with increasing frequency for all temperatures. Therefore, we can conclude that the mechanical response of a heavy-oil rock with changing temperature and frequency is strongly influenced by the behavior of the pore-filling oil.

\section{Modeling with a single aspect ratio}

Given that the measurements of the shear properties of the Uvalde oil and Uvalde rock are available, we use the fluid-substitution workflow described above. We first predict the effective elastic properties of the Uvalde rock saturated with the Uvalde oil using CPA. Then we compare the predicted storage modulus of the rock with laboratory data. We perform our modeling for the full range of frequencies and temperatures ranging from $30^{\circ} \mathrm{C}$ to $200^{\circ} \mathrm{C}$. We take the physical properties of the grain material as those of calcite $\left(K_{s}=60 \mathrm{GPa}, G_{s}=30 \mathrm{GPa}\right.$, density $\left.\rho_{s}=2.71 \mathrm{~g} / \mathrm{cm}^{3}\right)$. The dry shear modulus $G_{\text {dry }}$ of the Uvalde rock has not been measured, so we analyze the available values of the temperature- and frequency-dependent shear properties of the oil and rock to obtain $G_{\mathrm{dry}}$. In our analysis, we assume that at high temperatures and low frequencies, the pore fluid behaves as a Newtonian fluid and has no effect on the effective shear properties of the rock. Indeed, careful analysis of laboratory data (Figure 3 ) shows that for temperatures over $120^{\circ} \mathrm{C}$, the shear modulus of the Uvalde rock is a real constant $G=1.45 \mathrm{GPa}$. This means the effective shear modulus $G$ becomes identical to $G_{\text {dry }}$.

Next, we solve equations 3 and 4 to obtain $\alpha$ using the estimated $G_{\text {dry }}$. Then we use the forward CPA to compute the frequency- and temperature-dependent effective shear modulus of the Uvalde rock 
saturated with the Uvalde oil. In Figure 4a, we compare the calculated effective shear moduli of the rock (symbols) with the laboratorymeasured moduli (lines). We can see that CPA is unable to capture the trend of the frequency and temperature dependency of the rock shear properties, especially for low temperatures. The estimated moduli are significantly lower than the measured ones.

In Figure 4b, we compare the predictions of the effective storage modulus computed by the viscoelastic extension of Gassmann theory (EG) (Ciz and Shapiro, 2007) with laboratory data. The EG predictions (symbols) deviate from the laboratory data (lines) even more than the CPA estimates (Figure 4a).

\section{Compliant porosity}

For additional insight into frequency- and temperature-dependent behavior of Uvalde rock, we compare storage moduli of the rock and oil as a function of temperature for different frequencies (Figure 5).
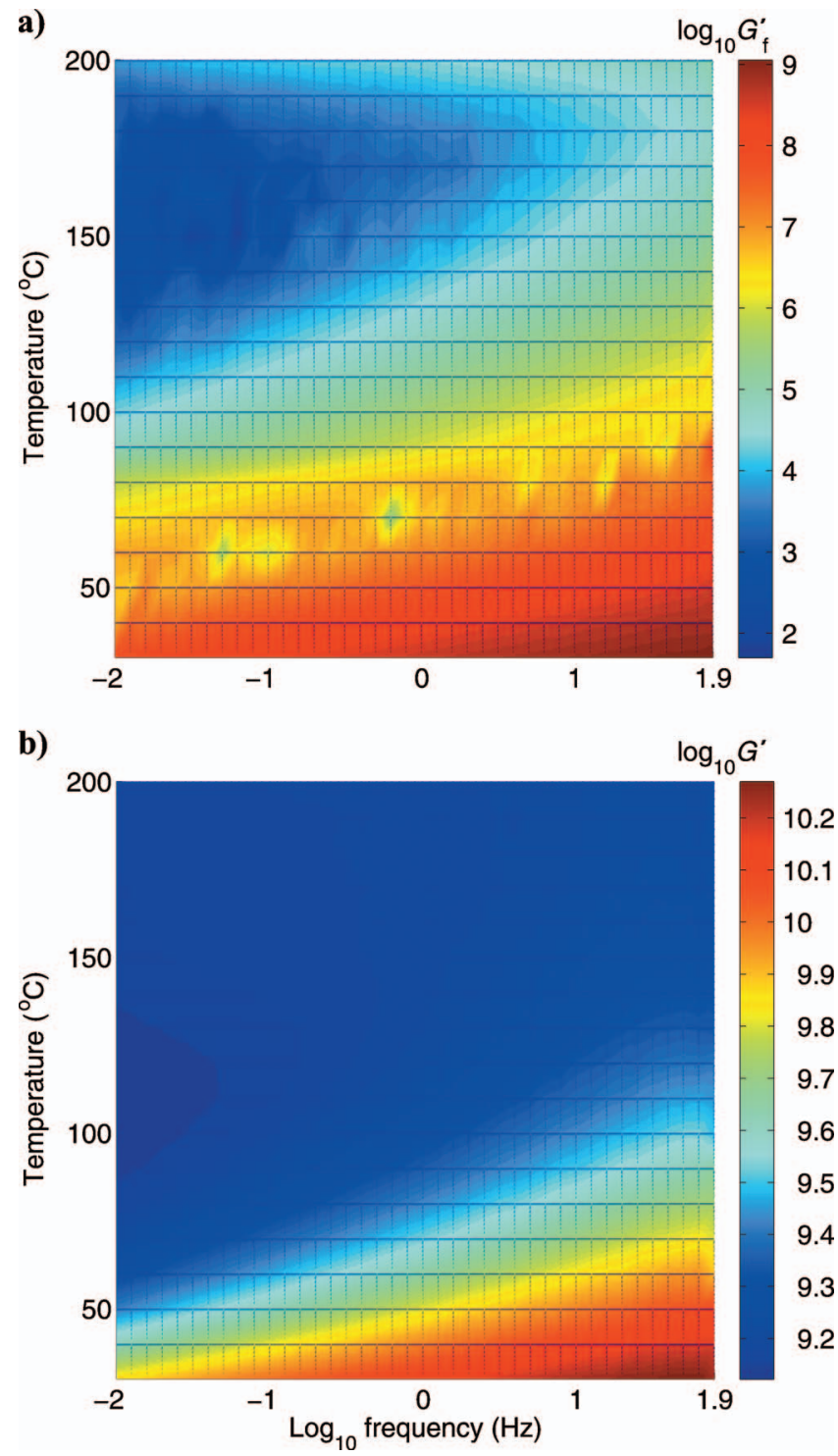

Figure 3. Variation of (a) storage modulus $G_{f}^{\prime}$ of Uvalde heavy oil and (b) storage modulus $G^{\prime}$ of Uvalde heavy-oil rock with frequency and temperature as measured by Behura et al. (2007).
We observe a substantial variation of the rock storage modulus $G^{\prime}$ (symbols) with temperature for different frequencies, contrary to the relatively moderate variation of the oil storage modulus $G_{f}^{\prime}$ (lines). For instance, $G^{\prime}(80-\mathrm{Hz}$ frequency) varies from approximately $2 \mathrm{GPa}$ up to $17 \mathrm{GPa}$, whereas $G_{f}^{\prime}$ varies from almost 0 to $1.2 \mathrm{GPa}$. This suggests that a simple mixing law, such as CPA with a single aspect ratio, cannot account for those observations.

The effect of porosity and fluids on elastic properties of rocks is often modeled using the binary structure of the pore space - relatively stiff pores that occupy most of the pore space and relatively compliant (soft) pores present at grain contacts, responsible for the pressure dependency of the elastic moduli. At high frequencies, fluid flow does not have enough time to equilibrate pore-pressure gradients between the two pore types, effectively isolating compliant pores from stiff pores and from each other. If a rock is saturated with viscoelastic fluid, this effect may occur even at relatively low frequencies, as considered in this study. When heavy oil is in a near-
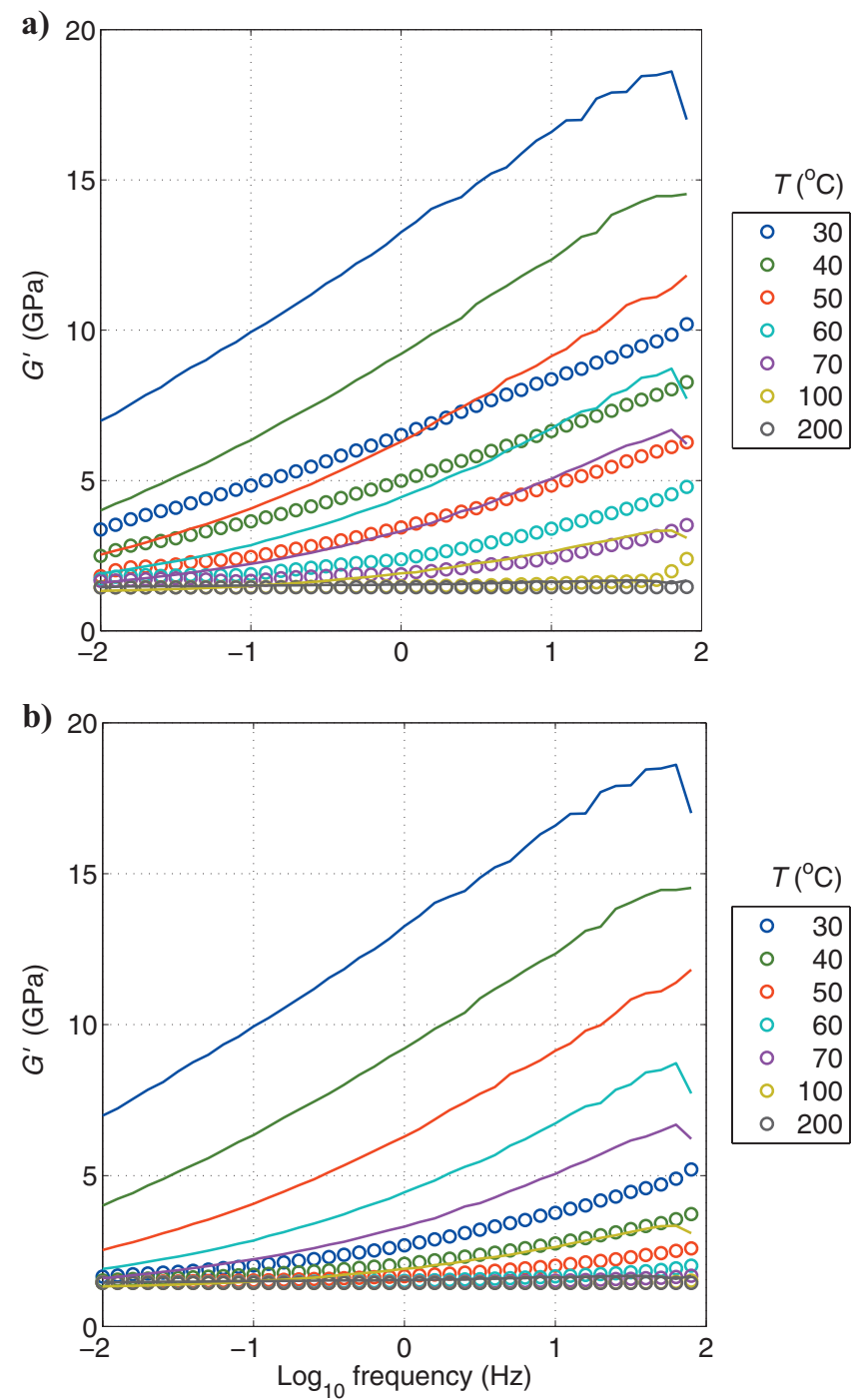

Figure 4. Comparison of frequency- and temperature-dependent storage modulus $G^{\prime}$ predicted by (a) CPA with a single aspect ratio (symbols) and by (b) extended Gassmann (symbols) with laboratory measurements conducted on a Uvalde heavy-oil rock sample (lines). $T$ is temperature. 
elastic state, it cannot flow out of soft pores, stiffening the compliant grain contacts. The presence of compliant pores may lead to additional dispersion of compressional and shear waves in heavy-oil rocks because of the dependency of the normal stiffness of these contacts on the storage modulus of the pore fill (Mavko and Jizba, 1991). Poor performance of both CPA with a single aspect ratio and EG may be explained by the presence of compliant pores in the rock matrix.

\section{Modeling with two aspect ratios}

To account for the effect of double porosity, we introduce the compliant porosity terms to equations 3 and 4 :

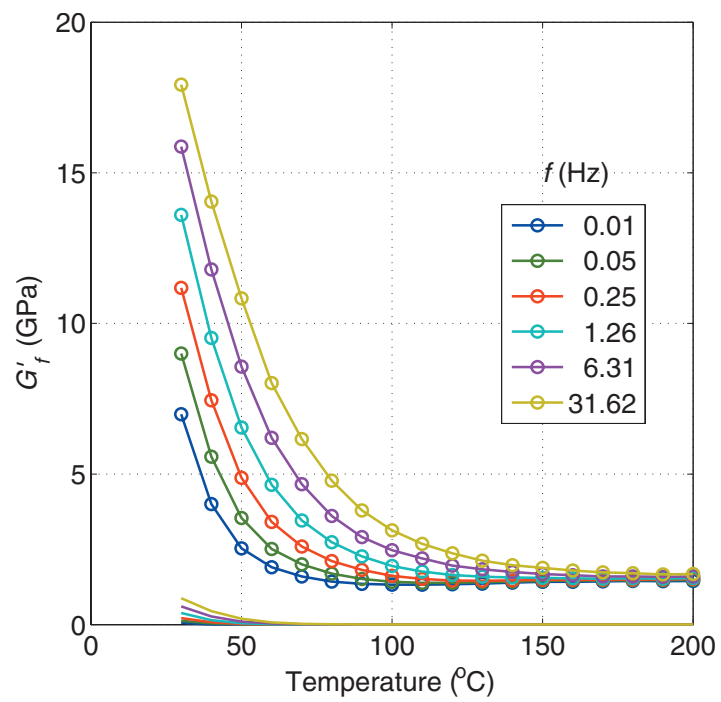

Figure 5. Laboratory measurements of storage moduli $G^{\prime}$ of Uvalde heavy oil (lines) and Uvalde heavy-oil rock (lines with symbols) for a range of frequencies and temperatures.

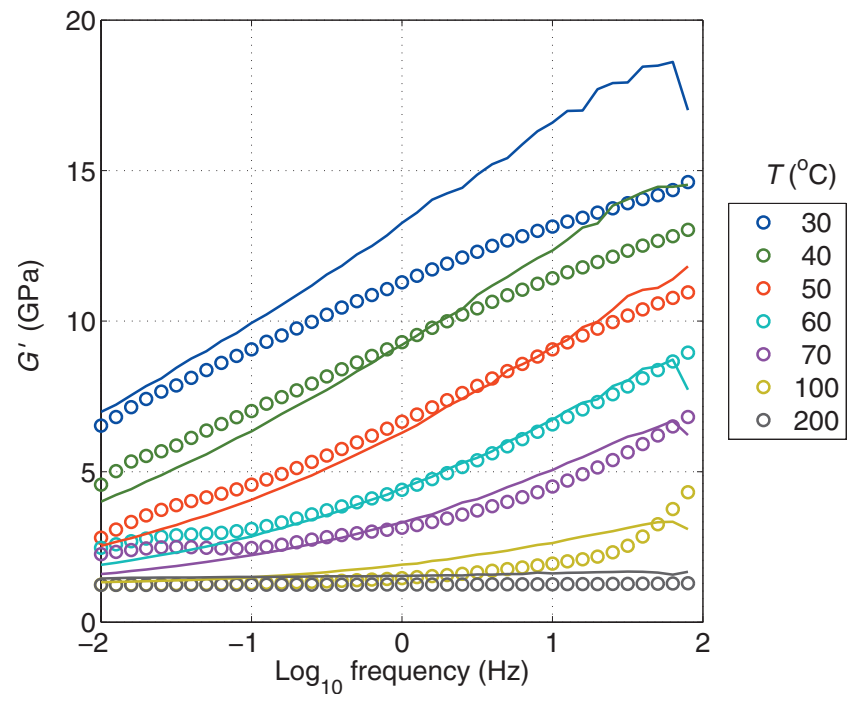

Figure 6. Comparison of frequency- and temperature-dependent storage modulus $G^{\prime}$ predicted by CPA with two aspect ratios (symbols) with laboratory measurements carried out on a Uvalde heavyoil rock sample (lines).

$$
\begin{aligned}
K_{n+1} & =\frac{\phi_{s} K_{f} P_{n}^{f s}+\phi_{c} K_{f} P_{n}^{f c}+(1-\phi) K_{s} P_{n}^{s}}{\phi_{s} P_{n}^{f s}+\phi_{c} P_{n}^{f c}+(1-\phi) P_{n}^{s}}, \\
G_{n+1} & =\frac{\phi_{s} G_{f} Q_{n}^{f s}+\phi_{c} G_{f} Q_{n}^{f c}+(1-\phi) G_{s} Q_{n}^{s}}{\phi_{s} Q_{n}^{f s}+\phi_{c} Q_{n}^{f c}+(1-\phi) Q_{n}^{s}},
\end{aligned}
$$

where $\phi_{s}$ is stiff porosity that occupies most of the pore space, $\phi_{c}$ is compliant porosity present within the grains and at grain contacts, and $\phi=\phi_{s}+\phi_{c}$.

Now the problem becomes more complicated because equations 6 and 7 must be inverted to estimate three unknown parameters: compliant porosity $\phi_{c}$, and aspect ratios of compliant and stiff pores $\alpha_{c}$ and $\alpha_{s}$, respectively. For the fitting procedure, we use dry shear modulus $G_{\text {dry }}$ and saturated shear modulus $G$ measured at $50^{\circ} \mathrm{C}$ and two frequencies, 0.08 and $15.85 \mathrm{~Hz}$. We assume the grains can be described by the aspect ratio $\alpha_{g}=0.8$ and keep it constant. The results of the fitting or the inverse CPA are $\phi_{c}=0.1, \alpha_{s}=1$, and $\alpha_{c}$ $=0.004$.

Next, we use the estimated parameters in the forward CPA scheme to predict the frequency- and temperature-dependent shear modulus $G$ of the Uvalde rock. The results are compared with measurements in Figure 6. We observe very good agreement between the CPA predictions (symbols) and the laboratory data (lines). The only deviation larger than the measurement error (approximately $\pm 2.09 \mathrm{GPa}$; see Behura et al., 2007) is observed at high frequencies for $30^{\circ} \mathrm{C}$.

We also compute the shear quality factor $Q$ (inversely proportional to the attenuation coefficient) from the predicted complex effective shear modulus of the rock. Figure $7 \mathrm{~b}$ shows $Q$ predicted by CPA. The general trend of estimated attenuation is similar to the measured one (Figure 7a). However, the measured $Q$ exhibits more significant variation with frequency.

\section{DISCUSSION}

The method described in this paper as well as other methods based on effective-medium theories are based on analogy between elasticity and viscoelasticity. This analogy is limited to sufficiently low frequencies such that the inertial terms in the equations of viscoelasticity are small and can be neglected (Hashin, 1970). This limitation is expected to be satisfied at seismic frequencies but may no longer be valid at higher frequencies where effects of squirt may become important (Leurer and Dvorkin, 2006).

We have described the CPA fluid-substitution workflow assuming a single aspect ratio. One can use a single aspect ratio when the range of pore shapes does not vary significantly. This might be the case when compliant grain-to-grain contacts or intragranular porosity does not significantly contribute to overall rock stiffness - for instance, well-consolidated rocks with welded grains or at high effective pressure when compliant pores are closed. However, in most sedimentary rocks, compliant pores (grain-to-grain contacts) with low aspect ratio contribute to the total porosity and are responsible for the stress dependency of elastic properties. When compliant pores are present in the rock matrix, introducing a second pore aspect ratio into the scheme becomes important.

Present work assumes that the porosity and geometry of the pore space of the rock matrix are the same for dry and oil-saturated rock, regardless of oil rheology. We also assume that oil composition does not vary with temperature. These conditions may be violated in field and laboratory studies (Behura et al., 2007; Han et al., 2007a). Note 

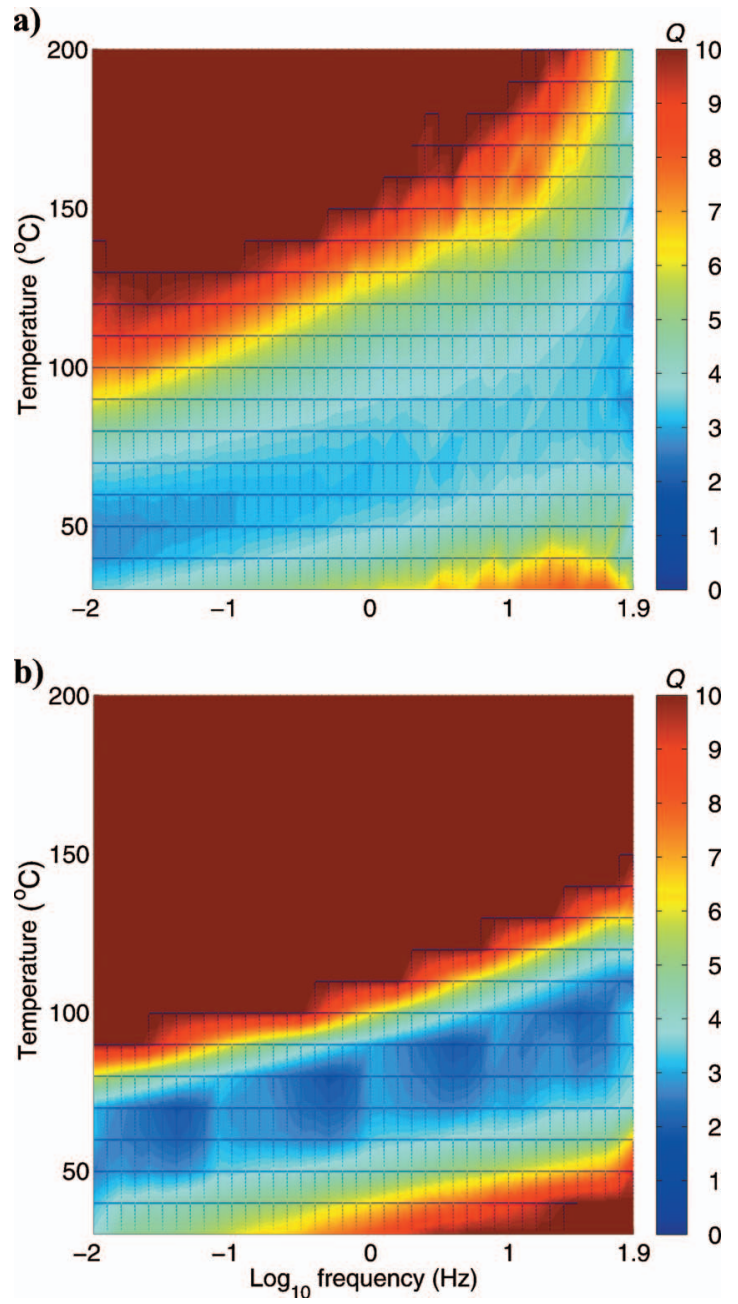

Figure 7. Variation of quality factor $Q$ of Uvalde heavy-oil rock with frequency and temperature (a) as measured by Behura et al. (2007) and (b) as predicted by CPA with two aspect ratios.

that we do not examine the pressure and gas effect on moduli/velocities, which might be important in the recovery process.

\section{CONCLUSIONS}

We have used a self-consistent mixing method known as coherent potential approximation as an alternative fluid-substitution scheme for rocks saturated with viscoelastic fluids. First, CPA equations were inverted to obtain effective aspect ratio of a rock from dry modulus. Then forward CPA with temperature- and frequency-dependent moduli of viscoelastic pore fill were used to obtain saturated moduli.

We tested CPA estimates of effective bulk moduli of a dry and a Newtonian fluid-saturated rock against analytical solutions. CPA predictions satisfy the Hashin-Shtrikman bounds and are approximately consistent with Gassmann equations and the Krief et al. model. A comparison with laboratory measurements revealed that CPA with a single aspect ratio is not adequate in predicting the frequency and temperature dependency of the effective shear modulus of the Uvalde heavy-oil rock.

We modified the scheme to account for the binary structure of the pore space. Inverted CPA was used to estimate three unknown pa- rameters: compliant porosity and aspect ratios of stiff and compliant pores from the laboratory-measured effective dry and saturated shear moduli at a single temperature and at two frequencies. In this case, CPA reproduced frequency- and temperature-dependent behavior of the shear modulus and attenuation of Uvalde heavy-oil rock, proven by good agreement with laboratory data. This confirms that the proposed scheme provides realistic estimates of the properties of heavy-oil rocks and can be used as an approximate fluid-substitution approach for rocks saturated with viscoelastic fluids.

\section{ACKNOWLEDGMENTS}

We thank the sponsors of the Curtin Reservoir Geophysics Consortium (CRGC), CSIRO Earth Science and Resource Engineering, and Australian Postgraduate Award (APA) for financial support. We also thank Konstantin Osypov, Oleg Dinariev, and Serge A. Shapiro for stimulating discussions.

\section{REFERENCES}

Batzle, M., R. Hofmann, and D.-H. Han, 2006, Heavy oils - Seismic properties: The Leading Edge, 25, 750-757.

Beggs, H. D., and J. R. Robinson, 1975, Estimation of the viscosity of crude oil systems: Journal of Petroleum Technology, 27, 1140-1141.

Behura, J., M. Batzle, R. Hofmann, and J. Dorgan, 2007, Heavy oils: Their shear story: Geophysics, 72, no. 5, E175-E183.

Berryman, J. G., 1980, Long-wavelength propagation in composite elastic media II. Ellipsoidal inclusions: Journal of the Acoustical Society of America, 68, 1980-1831.

- 1992, Single-scattering approximations for coefficients in Biot's equations of poroelasticity: Journal of the Acoustical Society of America, 91, 551-571.

Biot, M. A., 1956, Theory of propagation of elastic waves in a fluid saturated porous solid. II. Higher frequency range: Journal of the Acoustical Society of America, 28, 179-191.

Budiansky, B., 1965, On the elastic moduli of some heterogeneous materials: Journal of the Mechanics Physics of Solids, 13, 223-227.

Ciz, R., and S. A. Shapiro, 2007, Generalization of Gassmann's equations for porous media saturated with a solid material: Geophysics, 72, no. 6, A75A79.

Cole, K. S., and R. H. Cole, 1941, Dispersion and absorption in dielectrics, I - Alternating current characteristics: Journal of Chemical Physics, 9, 341-351.

Das, A., and M. Batzle, 2008, Modeling studies of heavy oil in between solid and fluid properties: The Leading Edge, 27, 1116-1123.

Eastwood, J., 1993, Temperature-dependent propagation of P-waves and S-waves in Cold Lake oil sands: Comparison of theory and experiment: Geophysics, 58, 863-872.

Ferry, J. D., 1980, Viscoelastic properties of polymers: John Wiley \& Sons, Inc.

Gassmann, F., 1951, Über die Elastizität poröser Medien: Vierteljahrsschrift der Naturforschenden Gesselschaft in Zürich, 96, 1-23.

Gurevich, B., 2002, Effect of fluid viscosity on elastic wave attenuation in porous rocks: Geophysics, 67, 264-270.

Gurevich, B., K. Osypov, R. Ciz, and D. Makarynska, 2008, Modeling elastic wave velocities and attenuation in rocks saturated with heavy oil: Geophysics, 73, no. 4, E115-E122.

Haddad, Y. M., 1995, Viscoelasticity of engineering materials: Springer

Han, D.-H., J. Liu, and M. Batzle, 2008, Seismic properties of heavy oils Measured data: The Leading Edge, 27, 1108-1115.

Han, D.-H., Q. Yao, and H.-Z. Zhao, 2007a, Complex properties of heavy oil sand: 77th Annual International Meeting, SEG, Expanded Abstracts, 1609-1613.

Han, D.-H., H.-Z. Zhao, and Q. Yao, 2007b, Velocity of heavy oil sand: 77th Annual International Meeting, SEG, Expanded Abstracts, 1619-1623.

Hashin, Z., 1970, Complex moduli of viscoelastic composites - I. General theory and application to particulate composites: International Journal of Solids and Structures, 6, 539-552.

Hashin, Z., and S. Shtrikman, 1963, A variational approach to the theory of the elastic behaviour of multiphase materials: Journal of the Mechanics and Physics of Solids, 11, 127-140.

Hill, R., 1965, A self-consistent mechanics of composite materials: Journal of the Mechanics and Physics of Solids, 13, 213-222.

Kazatchenko, E., M. Markov, and A. Mousatov, 2004, Joint modelling of 
acoustic velocities and electrical conductivity from unified microstructure of rocks: Journal of Geophysical Research, 109, B01202.

Krief, M., J. Garat, J. Stellingwerff, and J. Ventre, 1990, A petrophysical interpretation using the velocities of $\mathrm{P}$ and $\mathrm{S}$ waves (full-waveform sonic): The Log Analyst, 31, 355-369.

Küster, G. T., and M. N. Töksöz, 1974, Velocity and attenuation of seismic waves in two phase media - Part I: Theoretical formulations: Geophysics, 39, 587-606.

Leurer, K. C., and J. Dvorkin, 2006, Viscoelasticity of precompacted unconsolidated sand with viscous cement: Geophysics, 71, no. 2, T31-T40.

Marion, D., and A. Nur, 1991, Pore-filling material and its effect on velocity in rocks: Geophysics, 56, 225-230.

Mavko, G., and D. Jizba, 1991, Estimating grain-scale fluid effects on velocity dispersion in rocks: Geophysics, 56, 1940-1949.

Mavko, G., T. Mukerji, and J. Dvorkin, 1998, The rock physics handbook: Tools for seismic analysis in porous media: Cambridge University Press.

Milton, G. W., 1985, The coherent potential approximation is a realizable effective medium scheme: Communications in Mathematical Physics, 99, 463-500.

Mukerji, T., J. G. Berryman, G. Mavko, and P. A. Berge, 1995, Differential effective modelling of rock elastic moduli with critical porosity constraints: Geophysical Research Letters, 22, 555-558.

Nur, A., G. Mavko, J. Dvorkin, and D. Gal, 1995, Critical porosity: The key to relating physical properties to porosity in rocks: 65th Annual International Meeting, SEG, Expanded abstracts, 878-881.

Nur, A., C. Tosaya, and D. V. Thanh, 1984, Seismic monitoring of thermal enhanced oil recovery processes: 54th Annual International Meeting,
SEG, Expanded Abstracts, 118-121.

Ogushwitz, P. R., 1985, Applicability of the Biot theory. I — Low porosity materials: Journal of the Acoustical Society of America, 77, 429-440.

Pride, S. R., A. F. Gangi, and F. D. Morgan, 1992, Deriving the equations of motion for porous isotropic media: Journal of the Acoustical Society of America, 92, 3278-3290.

Schmitt, D. R., 1999, Seismic attributes for monitoring of a shallow heated heavy oil reservoir: A case study: Geophysics, 64, 368-377.

Smith, T. M., C. H. Sondergeld, and C. S. Rai, 2003, Gassmann fluid substitutions: A tutorial: Geophysics, 68, 430-440.

Thomsen, L., 1985, Biot-consistent elastic moduli of porous rocks: Low-frequency limit: Geophysics, 50, 2797-2807.

Tosaya, C., A. Nur, D. Vo-Thanh, and G. Da Prat, 1987, Laboratory seismic methods for remote monitoring of thermal EOR: SPE Reservoir Evaluation and Engineering, 2, 235-242.

Tsiklauri, D., and I. Beresnev, 2003, Properties of elastic waves in a nonNewtonian (Maxwell) fluid-saturated porous medium: Transport in Porous Media, 53, 39-50.

Williams, M. L., R. F. Landel, and J. D. Ferry, 1955, The temperature dependence of relaxation mechanisms in amorphous polymers and other glassforming liquids: Journal of the American Chemical Society, 77, 37013706.

Wu, T. T., 1966, The effect of inclusion shape on the elastic moduli of a twophase material: International Journal of Solids and Structures, 2, 1-8.

Xu, S., and R. E. White, 1995, A new velocity model for clay-sand mixtures: Geophysical Prospecting, 43, 91-118. 